\title{
Chroniques génomiques
}

\section{Perseverare}

diabolicum?

Bertrand Jordan

\section{IntegraGen récidive}

Le 5 avril dernier, l'entreprise française IntegraGen a annoncé [1] le lancement du test ARISk, « un outil génétique d'évaluation du risque d'autisme », commercialisé aux États-Unis au tarif de 2900 dollars. Nos lecteurs se souviendront d'une annonce très similaire, faite en 2005 , qui donna lieu à maints commentaires [2], à un procès en diffamation gagné par l'entreprise $[3,4]$ et constitua le point de départ d'un livre récemment paru $[5,6]$. En réalité, le test annoncé en 2005 n'a été commercialisé, aux États-Unis, qu'en 2011 et par les soins de la firme Transgenomic. Il a rencontré un succès si limité qu'il a été retiré du marché au bout de quelques mois.

Errare humanum est... mais l'entreprise persévère, on le voit. Il faut dire qu'à part d'importantes activités de service (séquençage à façon notamment) qui assurent l'essentiel de son chiffre d'affaires, l'autisme est le sujet principal d'IntegraGen aujourd'hui : I'apparition de cet « outil génétique» est donc logique. Vendu par la filiale américaine Integragen Inc., il repose sur l'analyse de « 65 marqueurs génétiques associés aux troubles du spectre autistique $»^{1}$, effectuée sur prescription médicale dans un laboratoire centralisé : il s'agit donc d'un test dit «LDT » (laboratory developed test) aussi familièrement appelé Home brew, catégorie qui (du moins jusqu'ici) ne requiert pas d'enregistrement par la FDA (food and drug administration). II est destiné à évaluer le risque pour des enfants frères ou sœurs

${ }^{1}$ Les chiffres et citations figurant dans ce paragraphe sont extraits du communique de presse d'IntegraGen diffusé le 5 avril 2012 [Arisk].

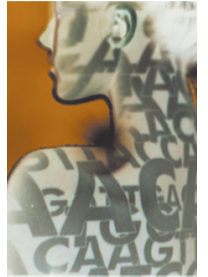

CoReBio PACA, case 901 , parc scientifique de Luminy, 13288 Marseille Cedex 9, France.

brjordan@club-internet.fr

d'enfants atteints et, selon le communiqué de presse, ceux qui sont identifiés comme à haut risque « ont un risque d'autisme de 2 à 4 fois plus important que le risque de récurrence évalué dans la population générale ». Rappelons qu'en 2005, et pour un test portant sur quatre marqueurs génétiques seulement, l'entreprise annonçait un risque relatif bien plus élevé, de 15. Comme le précise IntegraGen, les marqueurs génétiques en question sont des snip, et l'analyse différencie garçons et filles, suivant en cela une publication récente de l'entreprise [7] : 29 snip concernent uniquement les garçons, 28 les filles et 8 seulement sont étudiés chez les deux. Ce test « a été développé puis validé sur deux cohortes distinctes de patients représentant au total près de 2000 individus affectés de TSA ${ }^{2} \gg$.

Test plus évolué (65 marqueurs au lieu de 4), modestie dans l'annonce des performances, et présentation de l'analyse comme « un outil génétique d'évaluation du risque d'autisme » et non comme «le premier test génétique de l'autisme $»^{3}$ : il semble que les choses aillent dans le bon sens. Mais regardons de plus près la nature de ce test, et tentons de comprendre ses performances.

\section{Les bases d'un Genetic Score}

Comme lors des publications précédentes concernant un test à quatre snip [8], puis à huit snip [7], ce dernier

\footnotetext{
${ }^{2}$ TSA : troubles du spectre autistique.

${ }^{3}$ The world's first genetic test for autism, présentation relevée sur le site de l'entreprise le 22 juillet 2005, reproduite dans [5], figure 6, p. 36 .
} 
étant probablement identique à celui brièvement commercialisé par Transgenomic, le test actuel aboutit au calcul d'un score génétique d'après lequel sera évalué le risque de l'enfant. La différence est que, cette fois, les marqueurs (toujours des polymorphismes ponctuels) sont nombreux et différenciés selon le sexe. Les chiffres de prévalence et de risque moyen donnés dans le communiqué sont eux aussi différenciés: une prévalence de 1 sur 54 chez les garçons (ce qui semble vraiment très élevé, deux garçons sur cent, même s'il s'agit de TSA, c'est-à-dire d'autisme au sens large), de 1 sur 252 chez les filles; un risque de récurrence s'il y a déjà un enfant atteint qui est de 25,9\% chez les garçons, de 9,5\% chez les filles. Et si, comme indiqué dans le communiqué, les valeurs les plus élevées multiplient ce risque a priori par 4 , cela pourrait aboutir à une quasi-certitude dans certains cas! Pour aller plus loin, il faut s'appuyer sur les données non encore publiées par IntegraGen, mais présentées lors d'un congrès à Toronto le 17 mai, et dévoilées en partie dans un rapport technique, une sorte de publication non approuvée par un comité de lecture, mais disponible sur le site dédié au test [9]. On y apprend que le test a été étalonné sur une population de près de 2000 autistes au sens strict (critères étroits correspondant à une prévalence d'à peu près 1 sur 200) et de 400 témoins non autistes mais ayant un frère ou une sœur affecté. Le score génétique est calculé à partir des allèles trouvés pour les snip (aucun de ces allèles n'est rare dans la population) selon une formule qui revient à peu près à faire le total de tous les «mauvais » allèles; la valeur obtenue est comprise entre 27 et 56 pour les garçons, 29 et 54 pour les filles. Selon la valeur du score, on différencie cinq classes; celle dont le score est plus élevé correspond, sur cet échantillon, à un risque de $59 \%$ pour les garçons (donc le double environ du risque de récurrence a priori), et de $49 \%$ pour les filles, dont le risque a priori

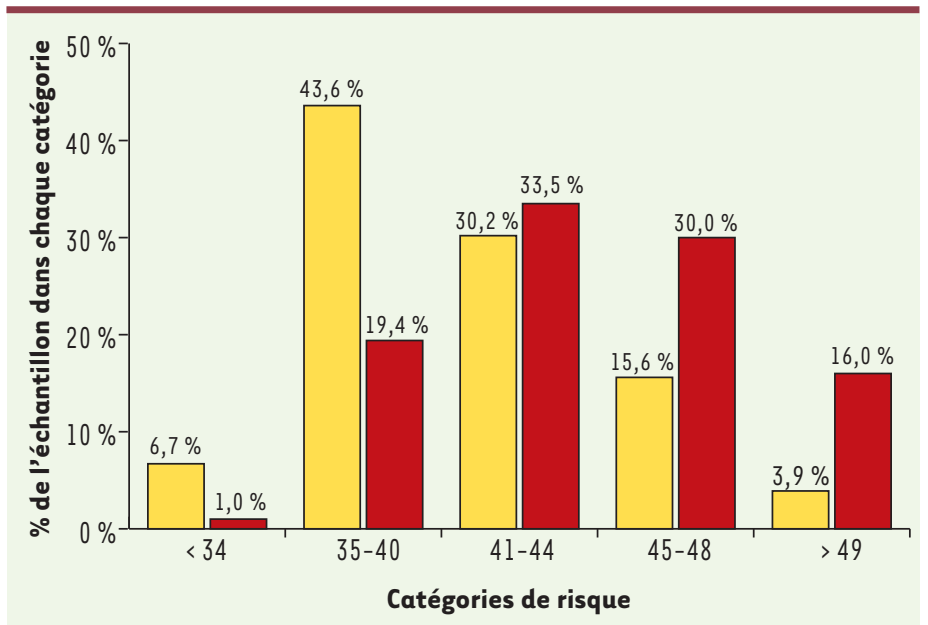

Figure 1. Résultats obtenus sur un échantillon de 1056 garçons diagnostiqués comme autistes (barres rouges) et de 179 garçons témoins (barres jaune). En ordonnée : pourcentage de l'échantillon retrouvé dans chaque intervalle de score génétique (total $=100 \%$ ). Les catégories de risque annoncées par IntegraGen sont, de gauche à droite, risque significativement diminué, modérément diminué, inchangé, modérément augmenté et significativement augmenté. Reproduction partielle et modifiée de la figure 2 de [8]. est de 9,5\%. La Figure 1 montre le résultat de l'emploi du test sur une population de plus d'un millier d'enfants diagnostiqués comme autistes et presque deux cents témoins. On voit que le score génétique est bien corrélé avec le diagnostic d'autisme (plus le score est élevé, plus la proportion d'autistes est forte), mais cette corrélation est loin d'être parfaite puisque seuls $46 \%$ des autistes sont classés comme à risque, catégorie qui englobe aussi presque 20 \% des témoins, et qu'un résultat symétrique est obtenu pour les scores faibles. Le test n'est donc qu'indicatif, comme le mentionne d'ailleurs l'entreprise.

On perçoit peut-être mieux l'utilité clinique du test (ou son absence) en considérant le rapport entre le risque « après le test » et le risque a priori, qui donne une idée du changement de perspective induit par la réalisation du test. Pour les garçons, ce rapport (risk ratio) va de 0,19 (pour la tranche de score la plus basse) à 2,28 (pour la plus élevée); pour les filles, de 0,42 à 4,69.

\section{L’utilité clinique}

La lecture d'un exemple de compte rendu, consultable sur le même site d'IntegraGen [10] permet de préciser les revendications de l'entreprise (Figure 2).

Concernant «John Doe » (Monsieur Dupont aux ÉtatsUnis), petit frère d'un autiste, et demandé par « John Smith, MD », il annonce pour ce patient un score génétique de 49 et précise que cela indique un risque de TSA de $59 \%$, multiplié par 2,28 par rapport au standard. Une liste des allèles trouvés pour les trente-sept snip examinés est donnée ainsi que des informations générales et une dizaine de publications. Mais, dès la première page, il est bien précisé que ce résultat ne peut « ni confirmer ni exclure un diagnostic de TSA », qu'il n'informe pas sur l'existence éventuelle d'une étiologie génétique spécifique (comme le syndrome de Rett ou celui de I'X fragile), et qu'il est recommandé d'effectuer un examen clinique par un spécialiste si le risque est élevé. Et le paragraphe se termine en donnant le numéro de téléphone d'un conseiller en génétique. En fait, comme déjà expliqué [5], l'utilité de ce test, selon ses vendeurs, est d'identifier des enfants à faire examiner en urgence par un vrai spécialiste de l'autisme afin, le cas échéant et si le diagnostic clinique est positif, de pouvoir entamer un traitement qui a d'autant plus de chance d'être efficace que le diagnostic est précoce. Compte tenu des performances somme toute modestes de cet «un outil génétique d'évaluation du risque d'autisme », on peut se demander si cela vaut réellement 2900 dollars américains. 


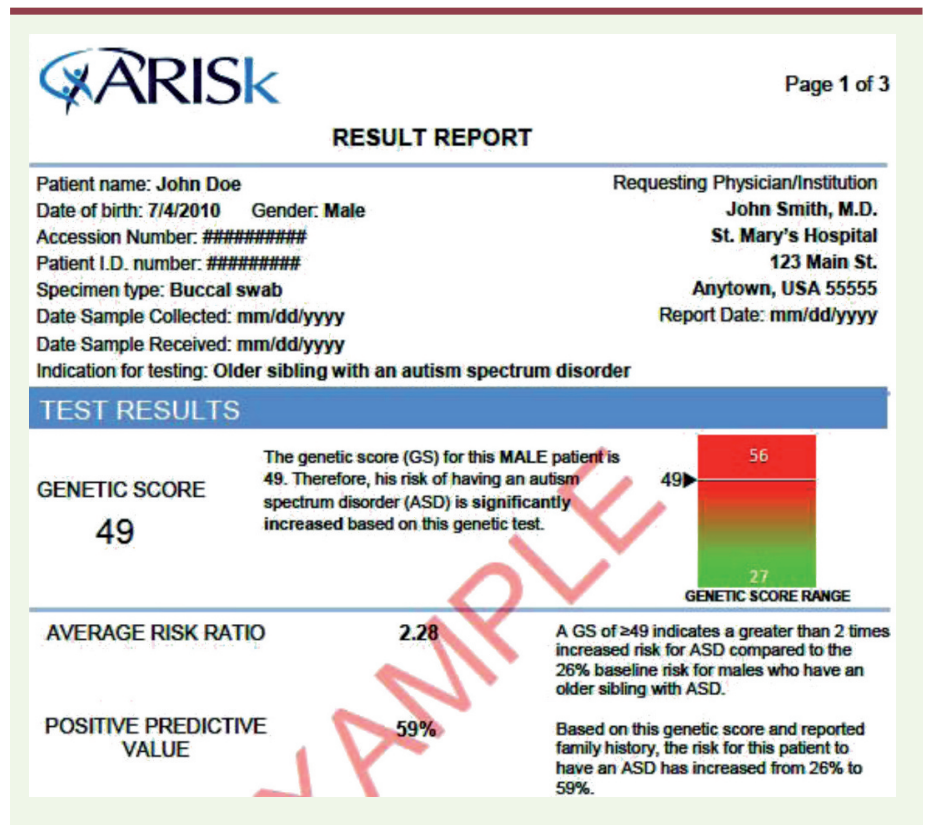

Figure 2. Vue partielle de la première page de l'exemple de résultat du test ARISk présenté sur le site consacré au test [9].

\section{Les aspects économiques et les nouveautés scientifiques}

D’une manière générale, les parents sont demandeurs d'un tel test - avec probablement des attentes irréalistes, l'ADN étant censé donner une réponse nette, en noir et blanc: nous avons vu que c'est loin d'être le cas. Selon un sondage pratiqué auprès d'un ensemble de pédiatres aux États-Unis, une petite moitié d'entre eux voit un intérêt majeur à ce test. Son coût élevé ne semble pas être un problème insurmontable : une législation déjà adoptée par plus de la moitié des États prévoit la prise en charge du traitement de l'autisme ${ }^{4}$, et un résultat positif du test permettrait aux parents de bénéficier plus tôt de ce soutien. Le remboursement de l'analyse serait déjà admis par plusieurs assureurs. L'entreprise voit donc l'avenir en rose : rappelons que le marché potentiel s'élève à plusieurs dizaines de milliers de tests par an rien qu'aux États-Unis. Le semi-échec de la version précédente de ARISk, commercialisé par Transgenomic en 2011, est attribué à ses performances plus faibles ( 8 snip en tout) et à des erreurs commerciales dans son ciblage. L'avenir nous dira ce qu'il en est.

Par ailleurs, tandis qu'IntegraGen peaufinait son test fondé sur les snip, la recherche a continué d'avancer et certaines nouveautés peuvent avoir un impact direct sur l'« outil génétique » proposé. Deux études récentes

${ }^{4}$ Par les approches comportementales, TEACCH ou ABA, coût de 35000 100000 dollars par an.
[11, 12] tendent à réviser à la baisse la composante génétique de l'autisme [13]. Elles portent toutes deux sur la comparaison de la concordance entre vrais et faux jumeaux. Rappelons que la très forte concordance entre vrais jumeaux (70 à $90 \%$ ), face à la faible valeur pour les faux jumeaux (10\%) constitue l'argument principal pour une forte héritabilité du syndrome. Or ces deux études donnent un écart nettement moins marqué, $47 \%$ versus $14 \%$ pour la première, $58 \%$ versus $21 \%$ pour la deuxième, ce qui abaisse la valeur de l'héritabilité, et naturellement diminue l'intérêt d'un « outil génétique $»^{5}$. Autre problème, le rôle qui se confirme de mutations de novo dans l'étiologie de l'autisme, mutations non détectées bien sûr par le test, et celui des CNV (copy number variation) $[14,15]$ sur lesquelles le test fait l'impasse. Et, bien sûr, on pourrait aussi penser à la séquence d'exomes [16] ou même de l'ADN entier, compte tenu des progrès incessants du séquençage de nouvelle génération.

\section{C'est « mieux »... mais est-ce « bien » ?}

Cet « outil génétique d'évaluation du risque d'autisme » représente sans nul doute un progrès par rapport au test esquissé en 2005. II est fondé sur un nombre assez important de snip, en accord avec ce que I'on sait aujourd'hui du caractère très multigénique de l'autisme, il a été testé sur un nombre respectable de patients et de témoins, la distinction qui est faite entre garçons et filles est intéressante, et les résultats sont nettement améliorés, même par rapport à la publication de 2010 [8]. Et, loin de revendiquer comme en 2005 un « test génétique de l'autisme », l'entreprise présente son produit comme un signal d'alerte pouvant suggérer un examen clinique approfondi, ce qui semble nettement plus raisonnable. On peut néanmoins rester sceptique sur l'intérêt réel de ce test, et même s'inquiéter de ses possibles effets négatifs. Compte tenu de ses performances modestes (Figure 1), cette analyse dont le coût reste élevé va-t-elle vraiment améliorer la prise en charge clinique précoce des autistes ? \&t, malgré la prudence dont fait (maintenant) preuve l'entreprise, le risque d'interprétations abusives est-il réellement écarté ? Pour le grand public, et pour beaucoup de médecins, un test génétique révèle un destin, ou à tout le moins une tendance forte - quelle va être l'attitude des parents et des soignants vis-à-vis des $20 \%$ d'enfants normaux (Figure 1) classés comme à risque plus élevé que la moyenne ? ARISk Test est dans l'air du temps, du moins outre-Atlantique, et sans doute va-t-il rencontrer un certain succès commercial - mais constitue-t-il réellement un progrès? II reste permis d'en douter. $\diamond$

\section{Perseverare diabolicum?}

\footnotetext{
${ }^{5}$ Assez curieusement, le premier travail conclut à l'absence d'influences environnementales partagées alors que le deuxième affirme leur importance. Voir notamment [13] pour une discussion détaillée de cette question.
} 


\section{LIENS D'INTÉRÊT}

L'auteur déclare n'avoir aucun lien d'intérêt concernant les données publiées dans cet article.

\section{RéFÉRENCES}

1. http://www.arisktest.com/60-press-releases.htm

2. Jordan B. Demandez le test de l'autisme ! Med Sci (Paris) 2005 ; 21 : 886-7.

3. Jordan B. L'autisme d'une entreprise. Med Sci (Paris) 2007 ; $23: 215-6$.

4. Jordan B, Favre M, Barthelemy $C$, et al. Génétique et business : défendons la liberté de critique! Med Sci (Paris) 2007 ; $23: 545-6$

5. Jordan B. Autisme, le gène introuvable. De la science au business. Paris : Seuil, 2012.

6. Gilgenkrantz S. Autisme, le gène introuvable. De la science au business (Bertrand Jordan). Med Sci (Paris) $2012 ; 28: 329-30$.

7. Carayol J, Schellenberg GD, Dombroski B, et al. Autism risk assessment in siblings of affected children using sex-specific genetic scores. Mol Autism $2011 ; 2$ : 17.

8. Carayol J, Schellenberg GD, Tores F, et al. Assessing the impact of a combined analysis of four common low-risk genetic variants on autism risk. Mol Autism 2010; $1: 4$.

9. Carayol J, Rio Frio T, Rousseau F, et al. Technical report: assessing genetic risk for autism spectrum disorder in siblings of children with autism using the ARISk ${ }^{\mathrm{TM}}$ Test. Accessible à partir de la page de description du test sur le site Arisktest.com, http://www.arisktest.com/62-developing-the-arisk-test.htm
10. http://www.arisktest.com/upload/pdf/Example\%20ARISk\%20 Test\%20Report.pdf.

11. Lichtenstein $P$, Carlström $\varepsilon$, Råstam M, et al. The genetics of autism spectrum disorders and related neuropsychiatric disorders in childhood. Am J Psychiatry 2010; 167 : 1357-63.

12. Hallmayer J, Cleveland $S$, Torres $A$, et al. Genetic heritability and shared environmental factors among twin pairs with autism. Arch Gen Psychiatry 2011 ; 68 : 1095-102.

13. Chamak B. L'autisme : surestimation des origines génétiques. Med Sci (Paris) $2010 ; 26: 659-62$.

14. Glessner JT, Wang K, Cai G, et al. Autism genome-wide copy number variation reveals ubiquitin and neuronal genes. Nature $2009 ; 459: 569-73$.

15. O’Roak BJ, Vives L, Girirajan S, et al. Sporadic autism exomes reveal a highly interconnected protein network of de novo mutations. Nature $2012 ; 485$ : 246-50.

16. Muers M. Human genetics: fruits of exome sequencing for autism. Nat Rev Genet $2012 ; 13: 377$

\section{TIRÉS À PART}

B. Jordan

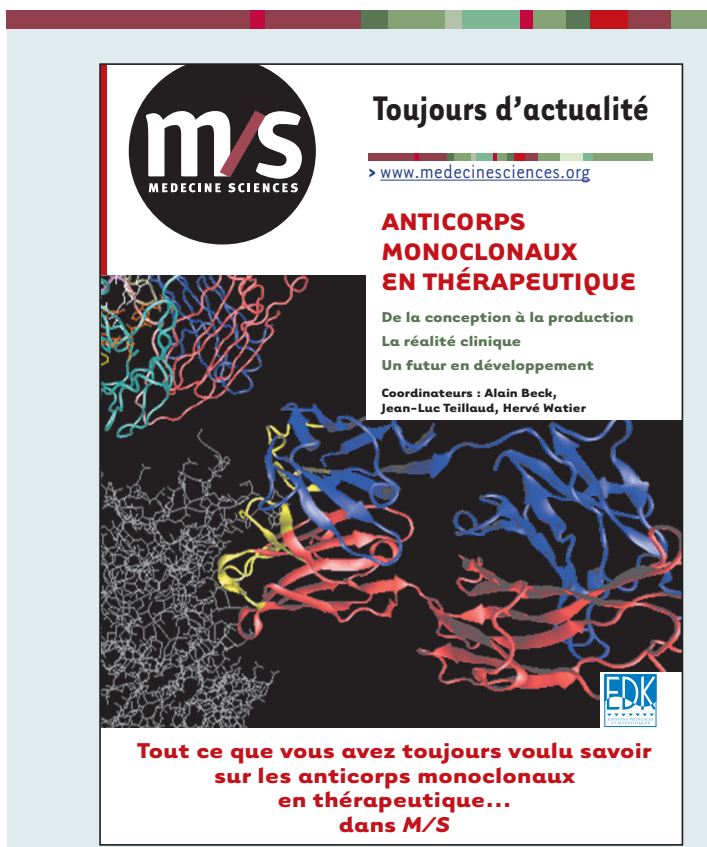

Tout ce que vous avez toujours voulu savoir sur les anticorps monoclonaux en thérapeutique... dans Médecine/Sciences. Pourquoi un numéro spécial de Médecine/Sciences sur les anticorps monoclonaux thérapeutiques? II nous a semblé que le moment était venu de dresser un état des lieux de ces biomédicaments qui prennent désormais une place considérable - et croissante dans les traitements de maladies souvent lourdes et désespérantes. Ce voyage que nous vous proposons à la découverte du monde des anticorps thérapeutiques nous a appris, ou plutôt rappelé, une évidence : les compétences en France sont fortes et nombreuses, qu'elles soient académiques ou industrielles, biotechnologiques ou cliniques. Le paysage français, trop longtemps discret, bruisse désormais de mille initiatives balayant de multiples aspects des anticorps thérapeutiques : études précliniques et cliniques menées avec de nouveaux anticorps dirigés contre des cibles originales, développement de nouveaux formats d'anticorps ou d'anticorps optimisés reposant sur des études structurales et fonctionnelles sophistiquées, recherche active de cibles pertinentes, mise au point de méthodologies de bioproduction, de couplage, etc. L'expansion industrielle rapide de ce champ est un défi que peut et doit relever notre pays, défi tant scientifique qu'économique, avec ses combats pour la propriété intellectuelle et pour l'emploi de nos jeunes scientifiques.

Alain Beck, Jean-Luc Teillaud, Hervé Watier

À retourner à EDK, 25, rue Daviel - 75013 Paris, France

Tél. : 0158101905 - Fax : 0143293262 - E-mail : edk@edk.fr

NOM :

Prénom :

Adresse :

Code postal :

Ville :

Pays :

Fonction :

Je souhaite recevoir $\mathrm{M} / \mathrm{S} \mathrm{n}^{\circ} \mathbf{1 2}$ - décembre 2009 (Anticorps monoclonaux en thérapeutique) : $25 €+3 €$ de port $=\mathbf{2 8} € \mathbf{T T C}$ en exemplaire, soit un total de $€$

$\square$ Par chèque, à l'ordre de $\mathbf{E} \mathbf{D}$ K Par carte bancaire : $\square$ Visa $\square$ Eurocard/Mastercard Carte $n^{\circ}$ Date d'expiration :

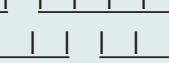

$\mathrm{N}^{\circ}$ de contrôle au dos de la carte

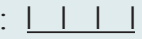

Signature : 